Proceedings of the $\bigcirc+\curvearrowleft$ Lake Louise Winter Institute

\title{
Fundamental Interactions
}


This page is intentionally left blank 


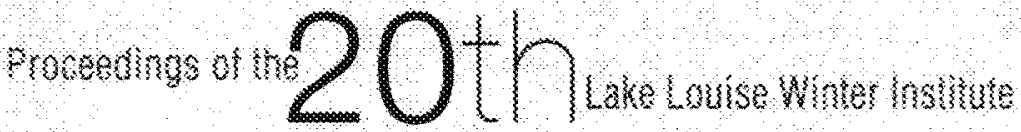

Fundamental

Interactions

Lakn' Loúse, Abseria, Canada . . 20-26 February 2005

editore

Alan Astbury

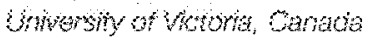

Aruce Campbell

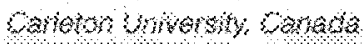

1.001\% Kramo

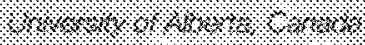

6.030. Moore

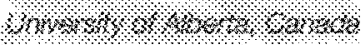

Hanwella Whoter.

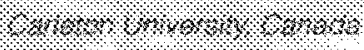


Published by

World Scientific Publishing Co. Pte. Ltd.

5 Toh Tuck Link, Singapore 596224

USA office: 27 Warren Street, Suite 401-402, Hackensack, NJ 07601

UK office: 57 Shelton Street, Covent Garden, London WC2H 9HE

\section{British Library Cataloguing-in-Publication Data}

A catalogue record for this book is available from the British Library.

\section{FUNDAMENTAL INTERACTIONS}

Proceedings of the Twentieth Lake Louise Winter Institute

Copyright $\odot 2006$ by World Scientific Publishing Co. Pte. Ltd.

All rights reserved. This book, or parts thereof, may not be reproduced in any form or by any means, electronic or mechanical, including photocopying, recording or any information storage and retrieval system now known or to be invented, without written permission from the Publisher.

For photocopying of material in this volume, please pay a copying fee through the Copyright Clearance Center, Inc., 222 Rosewood Drive, Danvers, MA 01923, USA. In this case permission to photocopy is not required from the publisher.

ISBN 981-256-631-7 


\section{PREFACE}

The twentieth Lake Louise Winter Institute, entitled Fundamental Interactions, was held from February 20-26, 2005 at the Chateau Lake Louise situated in the scenic Canadian Rocky Mountains. The format of the Winter Institute consisted of a mixture of pedagogical talks and short contributed presentations highlighting the latest results from experiments and new developments in theory. As usual, the sessions were held in the morning till noon and in the evening till at least 10:00 p.m. The participants had ample time for informal discussions in the afternoons or over meals. The afternoons were enjoyed by many for recreation and enjoyment of the winter wonderland in the Rockies.

The pedagogical talks focused on recent developments in cosmology. Results on K- and B-decays were critically assessed to bring out the new understanding of the topic. Results that lead to an unveiling of the new phases of QCD were presented. Physics with atomic traps and its impact on fundamental physics was clearly brought out. Finally the future experiments at the LHC and their discovery potential, in particular discovery of Higgs and possibly finding supersymmetric particles, was clearly spelled out. With the complement of contributed talks, a clear view of the present status of particle physics and cosmology was available.

We wish to thank Lee Grimard for a wonderful organisation of the Winter Institute with care, patience and skill. Our sincere thanks go to Suzette Chan for a masterful job of converting the contributions from the various participants into a nice proceedings. The support and help of the staff at the Chateau was available at all times, making it a rather smooth operation.

Finally we wish to thank the Deans of Science at the University of Alberta and Carleton University, the Institute of Particle Physics and TRIUMF for generous financial support. The Physics Department and Theoretical Physics Institute at the University of Alberta deserve a great deal of thanks for providing the infrastructural support that makes the task of arranging the Winter Institute much easier.

Organizing Committee

A. Astbury

B. A. Campbell

F. C. Khanna

R. W. Moore

M. G. Vincter 
This page is intentionally left blank 


\section{CONTENTS}

Preface $\quad v$

Contents vii

I. New Physics in $B$ and $K$ Decays 1

R. Fleischer

II. Physics at the Large Hadron Collider

M. Lefebvre

III. Applications of Trapped Atoms for Fundamental Symmetry Studies

G. Sprouse

Exclusive $D$ Semileptonic Decays at CLEO-c

N. E. Adam

Direct $C P$ Violation Results from $B_{A} B A R$

T. Allmendinger

Cosmic Ray Velocity and Electric Charge Measurements in the AMS Experiment

L. Arruda

Electroweak Physics at LEP 2

P. Azzurri

What Can We Learn about Neutrinos at SNOLAB?

A. Bellerive

Flavor and Chiral Oscillations with Dirac Spinors

A. E. Bernardini

Detection and Measurement of Gamma Rays in the AMS-02

Detector

J. Bolmont

Higgs Boson Discovery Potential at CMS

J. Brooke

AMS Transition Radiation Detector and the Search for

Dark Matter

G. Carosi 
viii

High Sensitivity B-Physics Measurements with the ATLAS

Detector

J. R. Catmore

Measurements of the CKM Angle $\alpha$ from $B A B A R$

C. A. Chavez

Systematics of Identified Hadron Spectra at PHENIX

M. Csanád

Deep, Dark \& Directional: The DRIFT Dark Matter

Experiment at Boulby Mine

J. C. Davies

Electroweak and QCD Results from DØ

M. Eads

Time Dependent CP Violation in $B^{0} \rightarrow \pi^{+} \pi^{-}$Decays

K. Hara

Modification of the Casimir Effect Due to a Minimal

Length Scale

U. Harbach

Exploring the Neutrino Universe with AMANDA and IceCube

D. Hardtke

Jet Production at HERA and Measurements of the Strong

Coupling Constant $\alpha_{s}$

D. Kcira

Parton Energy Loss, Saturation, and Recombination at BRAHMS

E.-J. Kim

Hadron Production and Radial Flow in $\mathrm{Au}+\mathrm{Au}$ Collisions at RHIC-PHENIX

A. Kiyomichi

SUSY Searches at LEP

A. C. Kraan

Search for New Physics at CDF II

A. Lath 
Search for Fermiophobic Higgs at LEP

V. Lemaitre

Measurements of Proton Structure at HERA

V. Lendermann

Top Physics at the LHC

S. Lowette

Spatial Confinement and Thermal Deconfinement in the Compactified Gross-Neveu Model

J. M. C. Malbouisson

Measurements of $\gamma$ in $B_{A} B A R$

G. Marchiori

Leptogenesis from Parametric Resonance

D. W. Maybury

Currents on Superconducting Strings in an unusual

Environment

M. A. Metlitski

Search for Technicolor at LEP

N. Meyer

Semileptonic Decays from $B A B A R$

A. K. Mohapatra

QCD Results at CDF

O. Norniella

Hadron Spectroscopy in Electrons-Protons Collisions at HERA

B. Olivier

Coulomb Corrections to R-Correlation in the Polarized Neutron Decay

A. Pak

Simulating the Sensitivity of $\mathrm{km}^{3}$ Hydrophone Arrays to Fluxes of Ultra High Energy Neutrinos

J. Perkin

DØ Top Physics

M. - A. Pleier 
DØ Higgs Physics Results

K. J. Rani

Measurements of $R_{b}$ at LEPII Energies

Y. Rozen

Quantization of Galilean Covariant Fields

E. S. Santos

Electroweak Measurements at CDF

A. Sidoti

Rare and Radiative $B$ Meson Decays from

the $B A B A R$ Experiment

J. Stelzer

The LHCb Ring-Imaging Cherenkov Detectors

J. W. Storey

Physics of Heavy Flavour at CDF

S. Torre

Time-Dependent $C P$-Violating Asymmetries in $b \rightarrow s \bar{q} q$ and $b \rightarrow s \gamma$ Transitions

Y. Ushiroda

Top Physics Results at CDF

T. Vickey

Inclusive Branching Fraction of $D \rightarrow v X$

M. Weinberger

Integral Fluxes, Day-Night, and Spectrum Results from SNO's 391-Day Salt Phase

J. Wendland

Jets and High $p_{T}$ (Di-Hadron) Correlations in PHENIX

D. L. Winter

Resonance Production at STAR

H. Zhang

List of Participants 